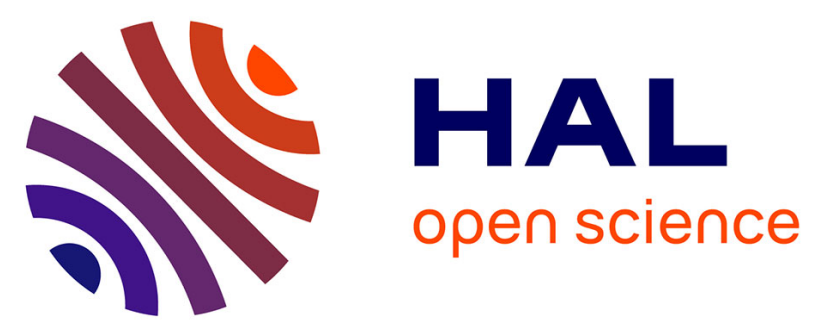

\title{
Neurophysiologie clinique en psychiatrie : 4 - Réalisation et interprétation des explorations du sommeil pour les pathologies du sommeil comorbides des troubles mentaux
}

Jean-Arthur Micoulaud-Franchi, Régis Lopez

\section{To cite this version:}

Jean-Arthur Micoulaud-Franchi, Régis Lopez. Neurophysiologie clinique en psychiatrie : 4 - Réalisation et interprétation des explorations du sommeil pour les pathologies du sommeil comorbides des troubles mentaux. Annales Médico-Psychologiques, Revue Psychiatrique, 2019, 177, pp.468 - 482. 10.1016/j.amp.2015.12.013 . hal-03480675

\section{HAL Id: hal-03480675 \\ https://hal.science/hal-03480675}

Submitted on 20 Dec 2021

HAL is a multi-disciplinary open access archive for the deposit and dissemination of scientific research documents, whether they are published or not. The documents may come from teaching and research institutions in France or abroad, or from public or private research centers.
L'archive ouverte pluridisciplinaire HAL, est destinée au dépôt et à la diffusion de documents scientifiques de niveau recherche, publiés ou non, émanant des établissements d'enseignement et de recherche français ou étrangers, des laboratoires publics ou privés.

\section{(c) (1) $\$$}

Distributed under a Creative Commons Attribution - NonCommerciall 4.0 International 


\section{Développement professionnel continu}

Neurophysiologie clinique en psychiatrie :

4 - Réalisation et interprétation des explorations du sommeil pour les pathologies du sommeil comorbides des troubles mentaux

Clinical Neurophysiology in Psychiatry:

\section{4 - Realization and interpretation of sleep exploration for sleep disorders with mental disorders comorbidities}

\section{Jean-Arthur Micoulaud-Franchi ${ }^{\text {a, }}$ * ${ }^{\text {, Régis Lopez }}{ }^{\text {c,d }}$}

a - Service d'Explorations Fonctionnelles du Système Nerveux, Clinique du Sommeil, CHU de Bordeaux, Place Amélie Raba-Léon, 33076 Bordeaux, France.

b - USR CNRS 3413 SANPSY, Université de Bordeaux, 33076 Bordeaux, France.

c - Centre National de Référence Narcolepsie Hypersomnie Idiopathique, Unité des Troubles du Sommeil, Hôpital Gui-de-Chauliac Montpellier, F-34000 France

d - Inserm U1061, Montpellier, F-34000 France

* Auteur correspondant : Dr Jean-Arthur Micoulaud-Franchi, Service d'explorations fonctionnelles du système nerveux, Clinique du sommeil, CHU de Bordeaux, Place Amélie Raba-Leon, 33076 Bordeaux, France.

Tel : 0622364019

Courriel : jarthur.micoulaud@gmail.com

\section{Résumé}

Les pathologies du sommeil de type troubles respiratoires du sommeil, mouvements anormaux liés au sommeil, comportements anormaux du sommeil (parasomnies), et hypersomnies centrales, sont fréquemment associées aux troubles psychiatriques. Il apparaît important de savoir les dépister afin d'orienter les patients si cela est nécessaire sur une exploration du sommeil adaptée. Les explorations du sommeil sont des examens paracliniques électrophysiologiques non invasifs essentiels pour apprécier le fonctionnement cérébral et plus généralement des grandes fonctions physiologiques cardiorespiratoire, motrice et comportementale au cours du sommeil. Ces examens permettent le diagnostic des pathologies du sommeil ainsi que l'évaluation de leur sévérité. L'objectif de cet article est premièrement d'apporter un minimum de connaissances aux psychiatres concernant la réalisation et 
l'interprétation des résultats des principales explorations du sommeil, et deuxièmement de rappeler les principes de dépistage des pathologies du sommeil comorbides en psychiatrie évaluées par ces explorations du sommeil. Une collaboration étroite entre médecine du sommeil et psychiatrie est nécessaire afin de favoriser l'accès pour les sujets souffrant de troubles psychiatriques aux explorations du sommeil dans l'objectif de leur assurer une prise en charge de qualité.

Mots clés: Neurophysiologie, Explorations du sommeil, Pathologies du sommeil, Comorbidité, Qualité de soins

\begin{abstract}
Sleep disorders like sleep related breathing disorders, sleep related movement disorders, parasomnias, and central hypersomnia are frequently associated with psychiatric disorders. It is thus important to detect patients with comorbid sleep disorders and to refer them for an appropriate sleep exploration if necessary. Sleep explorations are noninvasive electrophysiological diagnostic tests to assess brain function and more generally to assess the cardiopulmonary, motor and behavioral functions that are major physiologic functions modified during sleep. Sleep explorations allow diagnosis of sleep disorders and the assessment of their severity. The objective of this article is: i) to provide basic knowledge to psychiatrists on the conduct, the interpretation, and the results of the main sleep explorations, and ii) to highlight the principles of clinical screening of sleep disorders evaluated by the sleep explorations. Close collaboration between psychiatry and sleep medicine is needed to promote access to subjects suffering from mental disorder to sleep explorations with the aim to ensure quality of care.
\end{abstract}

Keywords : Neurophysiology ; Sleep explorations ; Sleep disorders ; Comorbidity ; Quality of Care

\title{
1 Introduction
}

Les explorations du sommeil sont des examens paracliniques électrophysiologiques non invasifs essentiels pour apprécier le fonctionnement cérébral et plus généralement des grandes fonctions physiologiques cardiorespiratoire, motrice et comportementale au cours du sommeil 
$[9,11,26]$. Ces examens permettent le diagnostic des pathologies du sommeil ainsi que l'évaluation de leur sévérité [3,9]. Les pathologies du sommeil sont fréquemment associées aux troubles psychiatriques [6]. Il s'agit donc de savoir dépister ces pathologies du sommeil comorbides afin d'orienter les patients si cela est nécessaire sur une exploration du sommeil adaptée [11].

L'objectif de cet article, dans la continuité des trois précédents articles sur la thématique de la neurophysiologie clinique en psychiatrie $[25,39,42]$, est premièrement d'apporter un minimum de connaissances aux psychiatres concernant la réalisation et l'interprétation des résultats des principales explorations du sommeil, et deuxièmement de rappeler les principales pathologies du sommeil comorbides en psychiatrie évaluées par ces explorations du sommeil [9]. Ne seront donc pas abordés dans cet article l'insomnie chronique [8,50] ainsi que les troubles du rythme circadien [12,36]. Ces pathologies du sommeil sont fréquemment associées aux troubles psychiatriques mais ne justifient qu'exceptionnellement la réalisation des explorations complémentaires du sommeil détaillées dans cet article [11,26].

\section{Les explorations du sommeil}

Les explorations traditionnellement réalisées en centre du sommeil permettent de recueillir des informations neurophysiologiques objectives sur l'architecture du sommeil mais également sur les fonctions cardiorespiratoires, motrices et comportementales modifiées pendant le sommeil [19].

\subsection{Description des examens traditionnels}

\subsubsection{Les signaux et paramètres enregistrés}

Les stades du sommeil sont évalués par un enregistrement électro-physiologique qui permet de recueillir pendant le sommeil du sujet les paramètres suivants [2, 41], Figure 1 :

- électro-encéphalogramme (EEG): activité électrique du cortex cérébral ;

- électro-oculogramme (EOG) : détection des mouvements oculaires ;

- électro-myogramme (EMG): mesure de l'activité musculaire.

\subsubsection{1 Électro-encéphalogramme}

Pour les principes généraux de l'enregistrement EEG et le vocabulaire de lecture de l'EEG, voir $[33,46]$. 
Dans le cadre des enregistrements de sommeil, l'EEG consiste à enregistrer l'activité électrique spontanée du cerveau, par l'intermédiaire d'électrodes placées sur le scalp du sujet, selon au moins trois dérivations d'avant en arrière [2], (Figure 1) :

- dérivations frontales : F4-M1 et F3-M2 ;

- dérivations centrales : C4-M1 et C3-M2 ;

- dérivations occipitales : O2-M1 et O1-M2.

Les dérivations sont donc enregistrées à droite $(\mathrm{F} 4, \mathrm{C} 4$ et $\mathrm{O} 2)$ et à gauche $(\mathrm{F} 3, \mathrm{C} 3, \mathrm{O} 1)$ non pas pour repérer des latéralisations de l'activité EEG du sommeil, mais pour des raisons de qualité de signal en cas d'artéfacts sur un côté. L'électrode de référence est systématiquement positionnée sur la mastoïde controlatérale (M1 pour les électrodes du scalp placées du côté droit et M2 pour les électrodes du scalp placées du côté gauche) afin d'obtenir une amplitude optimale du signal EEG par la plus grande distance inter-électrodes.

Le montage en dérivation antéropostérieure permet de repérer différentes figures neurophysiologiques du sommeil de topographie préférentiellement $[2,41]$ :

- frontale :

- complexes $\mathrm{K}$ : grandes ondes lentes avec une composante négative (vers le haut dans la nomenclature EEG) ample et rapide (mais $>0,5 \mathrm{~s}$ ), immédiatement suivie d'une composante positive moins ample et plus lente ;

○ ondes lentes du sommeil (dans la bande delta);

○ ondes en dents de scie (activité EEG dans la bande thêta en forme de dents de scie).

- centrale :

○ fuseau du sommeil (ou spindles) : activité EEG d'une fréquence de 12 à $16 \mathrm{~Hz}$ et d'une durée de quelques secondes (au moins une demi seconde);

○ pointes vertex : ondes pointues négatives de grande amplitude de durée courte $(<0,5 \mathrm{~s})$.

- occipitale :

- rythme alpha: activité EEG dans la bande de fréquence de 8 à $12 \mathrm{~Hz}$ disparaissant à l'ouverture des yeux et diffusant dans les régions antérieures en cas de somnolence.

\subsubsection{2 Électro-oculogramme}

L'EOG consiste à enregistrer les mouvements oculaires et palpébraux au moyen d'électrodes positionnées, pour l'une latéralement et au-dessus du canthus externe, et pour 
l'autre latéralement mais au-dessous du canthus interne (Figure 1). Ces électrodes sont référencées en M1 ou M2 [2]. Ce positionnement en opposition permet de différencier les figures neurophysiologiques du sommeil prédominant dans la région frontale (complexes $\mathrm{K}$ et ondes lentes) des mouvements oculaires et palpébraux. Les dérivations d'EOG légèrement décalées par rapport au plan médian permettent d'enregistrer les mouvements oculaires et palpébraux en opposition de phase alors que les figures neurophysiologiques du sommeil, qui peuvent diffuser sur les dérivations EOG, seront enregistrées dans la même phase.

L'électro-oculogramme permet de différencier trois types d'événements :

- les mouvements oculaires rapides (MOR), (Figure 2);

- les mouvements oculaires lents (MOL), d'une durée de quelques secondes, (Figure 2) ;

- les mouvements palpébraux, qui ont un aspect de pointes lentes de grande amplitude, (Figure 2).

\subsubsection{3 Électro-myogramme}

L'EMG consiste à enregistrer l'activité du muscle mentonnier considéré comme le témoin de l'activité musculaire au cours du sommeil [2]. (Figure 1). L'EMG mentonnier est enregistré avec trois électrodes dont les dérivations seront réalisées les unes par rapports aux autres. L'enregistrement EMG permet notamment de mesurer l'intensité de l'activité musculaire tonique et phasique pendant le sommeil. (Figure 2).

\subsubsection{Autres paramètres physiologiques du sommeil}

Les autres paramètres physiologiques recueillis pendant le sommeil du sujet sont [2], (Figure 1):

- les paramètres cardiorespiratoires (les débits respiratoires, la saturation en oxygène, les mouvements respiratoires thoraciques et abdominaux, le rythme cardiaque);

- les paramètres moteurs : détection des mouvements de jambes ;

- les paramètres comportementaux par enregistrement audio et vidéo.

Le débit respiratoire est évalué par une mesure indirecte de volume grâce à une mesure de la pression nasale via une canule nasale. Un capteur placé devant la bouche permet de s'assurer de la persistance ou pas d'un flux buccal grâce une thermistance sensible à l'air expiré plus chaud que l'air ambiant. La saturation en oxygène est évaluée par un oxymètre de pouls. Les mouvements thoraciques et abdominaux sont détectés par des sangles thoraciques et abdominales couplées à des capteurs piézoélectriques sensibles aux variations de forme. Le 
rythme cardiaque est évalué soit par l'oxymètre de pouls, soit par une dérivation électrocardiographique.

Les mouvements de jambes sont détectés par des électrodes positionnées sur les jambiers antérieurs droit et gauche.

Les comportements pendant le sommeil sont enregistrés par audio et vidéo (classique et infrarouge en condition d'obscurité) concomitantes et synchronisées aux autres paramètres mesurés. La description clinique des comportements sera rapportée et interprétée en fonction des autres paramètres physiologiques enregistrés dans une perspective électro-clinique classique en neurophysiologie [33, 46].

L'ensemble de ces paramètres permet le diagnostic :

- des troubles respiratoires du sommeil ;

- des mouvements anormaux liés au sommeil ;

- des comportements anormaux du sommeil ;

- des hypersomnolences et hypersomnies centrales, ainsi que leur sévérité.

\subsubsection{Les principales explorations du sommeil}

\subsubsection{La polysomnographie}

L'exploration complète du sommeil est la polysomnographie (PSG) [25] (Figure 1). Cet examen est habituellement réalisé dans les unités spécialement dédiées à l'exploration du sommeil. Ce type d'examen peut être réalisé à domicile [13, 14], avec des limites inhérentes à la qualité de l'enregistrement qui ne peut être contrôlée le long de sa réalisation et sur l'impossibilité de réaliser un enregistrement audio et vidéo simultanés (vidéopolysomnographie) qui renseigne sur les aspects comportementaux au cours du sommeil.

Une PSG enregistre simultanément tous les paramètres permettant d'apprécier la structure du sommeil (macro et micro-architecture), d'une part, et les événements pathologiques (respiratoires, moteurs et comportementaux), d'autre part. La PSG permet alors d'établir ou de confirmer le diagnostic de la plupart des pathologies du sommeil ainsi qu'en déterminer la sévérité [25]. Au moins huit types de signaux sont recueillis lors d'une PSG [1] :

- EEG ;

- EOG ;

- EMG mentonnier ;

- débits respiratoires ; 
- mouvements respiratoires thoraciques et abdominaux ;

- rythme cardiaque ;

- saturation en oxygène ;

- EMG des jambiers antérieurs ;

- $\quad+$ - position ;

- $\quad$ +/- ronflement ;

- $\quad$ +/- enregistrement audio-vidéo.

\subsubsection{La polygraphie ventilatoire}

La polygraphie ventilatoire (PV) est une exploration des paramètres cardiorespiratoires nocturnes. (Figure 1). Il s'agit d'un examen simplifié utilisé principalement pour le dépistage et le diagnostic du syndrome d'apnées du sommeil [1]. Il ne renseigne pas sur la quantité et la structure de sommeil, du fait de l'absence d'EEG, EOG et EMG. Il s'agit d'un examen plus facilement accessible que la PSG, utile en cas de probables syndrome d'apnées du sommeil en l'absence de suspicion d'autre pathologie du sommeil associée. L'enregistrement polygraphique comporte au moins quatre signaux :

- débit respiratoire ;

- mouvements respiratoires ;

- rythme cardiaque ;

- $\quad$ saturation en 02 ;

- $\quad$ +/- position ;

- $\quad+$ /- ronflement.

\subsubsection{Les mesures objectives de somnolence diurne}

Les Tests Itératifs de Latence d'Endormissement (TILE) [5] et les Tests de Maintien de l'Eveil (TME) [44] permettent d'évaluer de façon objective la plainte de somnolence diurne. Ils sont réalisés avec un montage comprenant des paramètres EEG, EMG et EOG [29]. Il est recommandé de s'assurer d'un sommeil adéquat via une PSG avant la réalisation d'un TILE ou TME.

Les Tests Itératifs de Latence d'Endormissement (TILE) mesurent la propension du sujet à s'endormir la journée. Le sujet, allongé dans un lit, a comme consigne lors de cinq sessions (généralement à 9,11, 13, 15 et 17 heures) : «Allongez vous calmement, fermez les yeux et essayez de dormir. » Le test est interrompu au bout de 20 minutes si le sujet ne s'est pas endormi ou 15 minutes après son endormissement [5]. 
Les Tests de Maintien de l'Eveil (TME) mesurent la capacité du sujet à rester éveillé durant la journée. Le sujet, installé dans un fauteuil en semi-pénombre, a comme consigne lors de quatre sessions (généralement à 10,12, 14 et 16 heures) : «Restez éveillé, luttez contre le sommeil le plus longtemps possible: gardez les yeux ouvert, regardez devant vous. » Le test est interrompu au bout de 40 minutes si le sujet ne s'est pas endormi [44].

\subsection{Interprétation des examens}

L'apparition des analyses automatiques du sommeil manque d'exactitude et n'a pas conduit à la disparition de la nécessité de l'analyse visuelle des tracés du sommeil [41]. L'analyse du sommeil demande donc un « œil clairvoyant », comme le soulignaient Gibbs et Gibbs en 1941 dans un des premiers atlas EEG [21]. Leur préface s'ouvrait ainsi : «Ce livre a été écrit dans l'espoir d'aider le lecteur à voir d'un coup d'œil ce que d'autres ont mis de nombreuses heures à trouver, et de l'aider à exercer son œil afin de parvenir à des diagnostics fondés sur des critères subjectifs » [16]. L'utilisation du terme «subjectif » peut surprendre et les auteurs de poursuivre : «Pour autant, déprécier l'usage [des] indices et mesures objectives serait une erreur : elles ont leur utilité et doivent être mobilisées le plus souvent possible. Mais «l'œil clairvoyant» (seing eye) issu d'une parfaite familiarité avec le matériel est l'instrument le plus précieux que puisse posséder l'électroencéphalographiste; personne ne saurait être vraiment compétent avant de l'avoir acquis » [16]. En 1950, dans la nouvelle édition de leur atlas, la préface rajoutait: «L'expérimentation sur les ondes est importante [...] et avec l'analyse fréquentielle de l'électroencephalogramme [...] elle montre qu'un indice objectif n'égale l'exactitude de l'évaluation subjective. [...] L'exactitude ne doit pas être sacrifiée à l'objectivité, [...] l'analyse doit être menée comme une opération intellectuelle plutôt qu'électromécanique » [16].

Il en est de même pour l'exploration du sommeil qui nécessite un codage visuel des stades du sommeil et des événements dont l'apprentissage expertal a permis une opération intellectuelle adéquate. Les mesures objectives, «électromécaniques » ou automatiques du sommeil manqueraient d'exactitude et seraient sacrifiées à l'objectivité prétendue de ces mesures. Pour autant, l'analyse visuelle du sommeil conduit à des index et scores objectifs.

Il est ainsi intéressant de noter que l'analyse du sommeil soulève des questionnements proches de ceux de la sémiologie psychiatrique, en distinguant deux approches : l'une, intuitive, basée sur une appréhension globale de la symptomatologie et l'autre, analytique, basée sur une recherche de critères sémiologiques [34]. Ces deux approches sont 
complémentaires, et la première, expertale, permet un diagnostic rapide qui peut renvoyer à la seconde en cas de difficultés. Bien que les tracés du sommeil puissent s'appréhender de manière globale avec l'expérience, une approche critériologique internationalement reconnue a été construite et doit être suivie [2].

\subsubsection{La structure du sommeil}

La macro-architecture du sommeil correspond à l'organisation générale du sommeil, définie selon une analyse de l'enregistrement par périodes de 30 secondes (époques). La représentation visuelle de la macro-architecture du sommeil s'appelle l'hypnogramme [19].

Les stades de sommeil sont définis visuellement en fonction de la présence des différentes figures physiologiques du sommeil évaluées en EEG, EOG et EMG. Il s'agit d'attribuer un état et un seul à chaque époque. Si plusieurs états sont présents, celui qui occupe plus de $50 \%$ de la durée de l'époque est retenu [2].

Ainsi les différents états (veille et stades de sommeil) sont définis selon les critères suivants [2] (Figure 2) :

- la veille $(\mathrm{W})$ : elle correspond à deux états physiologiques.

○ La veille active : les yeux sont ouverts, on observe de nombreux mouvements oculaires et palpébraux. L'activité EEG de fond est rapide et de bas voltage (dans bande béta), associée à des artefacts musculaires nombreux.

- La veille calme : l'activité EEG de fond est principalement caractérisée par la présence de rythme alpha. Les yeux sont fermés ou ouverts ;

- le stade N1 (NREM1) : l'activité EEG de fond est formée de fréquences mixtes (dans les bandes alpha et thêta), associée à de possibles pointes vertex et des MOL.

- le stade N2 (NREM2) : l'activité EEG de fond est mixte ou dans la bande thêta (avec moins de $20 \%$ de l'époque dans la bande delta), associée aux grapho-éléments caractéristiques (les complexes $\mathrm{K}$ et les fuseaux);

- le stade N3 (NREM3) : l'activité EEG de fond est caractérisée par la présence d'au moins $20 \%$ d'ondes lentes appartenant à la bande delta ;

- le stade REM : on distingue deux types d'activités au cours de ce stade. L'activité tonique est durable, elle associe un EEG de fond de fréquences mixtes (alpha et thêta) et une abolition du tonus musculaire. L'activité phasique est intermittente, elle associe de brèves contractions des extrémités (twitches), des MOR et un aspect d'ondes en dents de scie sur l'EEG. 
L'analyse de la macro-architecture du sommeil fournit des informations quantitatives et qualitatives sur la qualité générale du sommeil, définies selon les paramètres suivants :

- le temps passé au lit lumière éteinte (de la lumière éteinte le soir à la lumière allumée le matin) ;

- la période totale de sommeil (de la première époque de sommeil à la dernière époque de sommeil);

- la latence d'endormissement (de la lumière éteinte le soir à la première époque de sommeil), normalement inférieure à 30 minutes,

- la veille intra-sommeil (temps cumulé de veille entre la première et la dernière époque de sommeil);

- le temps total de sommeil (période totale de sommeil moins le temps de veille intrasommeil), normalement supérieure à six heures ;

- l'efficacité du sommeil (temps total de sommeil sur le temps passé au lit lumière éteinte), normalement autour de 80-90\% ;

- les pourcentages des différents stades de sommeil (temps cumulé passé dans un stade sur le temps total de sommeil);

- l'organisation cyclique du sommeil (un cycle est défini visuellement par la succession d'époques de stades NREM suivie d'une phase de stade REM);

- la composition des cycles de sommeil (analyse visuelle de la proportion des différents stades par cycle de sommeil);

- la latence d'apparition du rem (temps entre la première époque de sommeil et la première époque de REM).

La micro-architecture du sommeil apparaît après une analyse plus fine de l'enregistrement de sommeil. En pratique clinique courante, l'analyse micro-architecturale du sommeil consiste en la mesure de l'index de réactions d'éveil (nombre total de réactions d'éveil par heure de sommeil) [2]. Une réaction d'éveil est définie par une augmentation brusque de la fréquence de l'activité EEG de fond pour une durée de 3 à 15 secondes (au-delà de 15 secondes, l'époque est qualifiée en veille) (Figure 2, D). Elle peut être associée à une réaction autonomique (accélération du rythme cardiaque). L'index des réactions d'éveil est le reflet de la fragmentation du sommeil, spontanée ou induite par des événements extrinsèques environnementaux, et/ou pathologiques (respiratoires et moteurs).

D'autres paramètres micro-architecturaux peuvent être quantifiés, le plus souvent dans le cadre de la recherche (densité des fuseaux, analyse de l'activité à ondes lentes, quantification de l'activité musculaire tonique et phasique en SP). 
Les psychotropes utilisés en psychiatrie rendent souvent l'interprétation des tracés EEG de sommeil difficile. L'activité EEG est souvent modifiée, en particulier avec l'apparition de rythmes rapides (Figure 1). Par ailleurs, la macro-architecture du sommeil peut être perturbée, (pour revue voir [11]). Ainsi, un enregistrement de sommeil doit autant que possible être réalisé en l'absence de médication psychotrope. Le cas échéant, son interprétation doit prendre en compte l'effet de ses traitements sur le sommeil.

\subsubsection{L’index d'apnées hypopnées (IAH)}

Il existe deux types d'événements respiratoires [1,9,44,48] (Figure 3) :

- une apnée correspond à un arrêt complet du débit respiratoire pour une durée d'au moins dix secondes chez l'adulte ;

- une hypopnée correspond à une diminution du débit respiratoire d'au moins dix secondes chez l'adulte, associée à une désaturation en oxygène de plus de $3 \%$ et/ou à une réaction d'éveil.

La persistance ou non des mouvement respiratoires thoraciques et abdominaux permet de qualifier un événement respiratoire :

- d'obstructif, lorsque persiste un effort respiratoire avec l'apparition d'un déphasage des mouvements thoraciques et abdominaux (Figure 3);

- central, lorsqu'il existe une disparition des efforts respiratoires (Figure 3).

De plus, un capteur renseigne généralement sur la position du sujet, permettant de comptabiliser les événements respiratoires en fonction de la position dans laquelle ils surviennent.

Le compte rendu de l'exploration du sommeil indiquera l'index d'apnées hypopnées par heure du sommeil (IAH), le pourcentage d'apnées hypopnées obstructives et centrales, l'index de réactions d'éveil provoquées par les événements respiratoires, et le pourcentage d'événements en décubitus dorsal. L'index de désaturation en oxygène de plus de $3 \%$ sera également indiqué. Un IAH supérieur à $5 / \mathrm{h}$ est pathologique [1].

\subsubsection{L’index de mouvements périodiques des membres inférieurs (MPMI)}

Chaque contraction musculaire de 0,5 à 10 secondes enregistrée par les EMG positionnés sur les jambiers antérieurs est comptabilisée. Le caractère périodique d'un 
mouvement est défini par son appartenance à une série d'au moins quatre mouvements, séparés de 4 à 90 secondes [2] (Figure 3).

Le compte rendu de l'exploration du sommeil indiquera l'index de MPMI par heure de sommeil et l'index de réactions d'éveil provoquées par les mouvements. Un index de MPMI supérieur à 15/h est pathologique s'il est associé à des plaintes du sujet.

\subsubsection{Les comportements anormaux pendant le sommeil}

L'enregistrement vidéo permet d'identifier des comportements anormaux pendant le sommeil et d'en diagnostiquer l'étiologie [45]. Une attention particulière doit être apportée à deux types de comportements moteurs anormaux au cours du sommeil :

- en sommeil lent profond peuvent survenir lors de transitions entre le sommeil lent profond (N3) et la veille, des comportements plus où moins complexes d'allure confus, associés à une persistance de l'activité à ondes lentes (appelée «hypersynchronie d'éveil») plus ou moins accompagnés d'une hyperactivation autonomique (accélération du rythme cardiaque) (Figure 4);

- en sommeil paradoxal peuvent survenir des comportements associant des secousses musculaires, des mouvements brusques plus ou moins élaborés, accompagnés parfois de somniloquie. Ces comportements sont caractérisés à l'EMG par une reprise brutale du tonus musculaire, et sont accompagnés d'une augmentation générale de l'activité musculaire phasique en REM.

Il faut également savoir distinguer des comportements stéréotypés, parfois latéralisés, avec ou sans composante tonico-clonique, en général multiples sur l'enregistrement, et survenant sans préférence de stade de sommeil, qui peuvent faire évoquer une épilepsie frontale nocturne [43].

Le compte rendu de sommeil indiquera la survenue ou non de comportements anormaux au cours du sommeil.

\subsubsection{Les mesures de la somnolence diurne}

Le TILE est une mesure de la somnolence permettant de quantifier l'aptitude et la manière de s'endormir, alors que le TME est une mesure de la somnolence permettant de quantifier l'aptitude à lutter contre l'endormissement, cette mesure étant reliée au risque accidentel secondaire à la somnolence diurne [4]. 
Le TILE recueille deux paramètres, la latence moyenne d'endormissement (moyenne du délai d'apparition de la première époque de sommeil sur les cinq tests) et la latence d'apparition du stade REM pour chaque test. Une apparition d'un stade REM inférieure à 15 minutes signe un endormissement en sommeil paradoxal. Une latence moyenne d'endormissement inférieure à 8 minutes est pathologique. Le TILE est un outil diagnostique. Il permet d'objectiver le caractère pathologique ou non d'une plainte d'hypersomnolence, il est nécessaire au diagnostic des hypersomnies centrales [5].

Le TME recueille la latence moyenne d'endormissement (moyenne du délai d'apparition de la première époque de sommeil sur les quatre tests). Une latence d'endormissement moyenne inférieure à 19 minutes est pathologique, signant une difficulté pour le sujet à maintenir un éveil suffisant lorsqu'il n'est pas stimulé [44]. Contrairement au TILE, le TME n'est pas un test diagnostique mais une mesure de l'efficacité de la prise en charge de la somnolence diurne, quelle qu'en soit l'étiologie. Son interprétation doit être rigoureuse car elle revêt un caractère médicolégal, ce test autorisant ou non les conducteurs professionnels traités pour leur somnolence diurne à la reprise de la conduite automobile (arrêté du 31 août 2010) [37].

La sévérité de la somnolence diurne peut être également appréciée de manière subjective au moyen d'outils cliniques simples comme l'échelle de somnolence d'Epworth qui est un auto-questionnaire qui peut être facilement complété à la consultation [8]. Un score supérieur à 10/24 indique une somnolence diurne excessive, celle-ci étant sévère au-delà de 15/24 (Tableau 1). Cette échelle mesure principalement les conséquences fonctionnelles de la somnolence diurne. Ainsi, la sévérité de la somnolence diurne mesurée électrophysiologiquement par le TILE et le TME n'est que faiblement corrélée aux scores de l'échelle de somnolence d'Epworth [22]. De ce fait, une somnolence subjective d'allure pathologique doit systématiquement être confirmée par des mesures électrophysiologiques objectives. Ceci est d'autant plus vrai pour l'évaluation de la réponse thérapeutique via les TME du fait des implications médicolégales.

\section{Les troubles du sommeil en psychiatrie}

Les pathologies du sommeil pouvant bénéficier d'une exploration du sommeil détaillée précédemment concernent :

- la respiration avec les troubles respiratoires du sommeil ;

- la motricité avec les mouvements anormaux liés au sommeil ; 
- les comportements anormaux du sommeil avec les parasomnies ;

- la somnolence diurne avec les hypersomnolences et les hypersomnies centrales.

\subsection{Les troubles respiratoires du sommeil}

\subsubsection{Généralités}

Le trouble respiratoire du sommeil le plus fréquent est le syndrome d'apnées obstructives du sommeil (SAOS) [3,24]. L'ancien syndrome de résistance des voies aériennes supérieures (SARVAS) est désormais intégré dans le SAOS. Le diagnostic de SAOS doit être évoqué devant :

- des symptômes diurnes (somnolence diurne, sommeil perçu comme non réparateur, céphalées matinales, plaintes cognitives, baisse de la libido, symptômes dépressifs et irritabilité) ;

- des manifestations nocturnes (ronflement, sensations de suffocation nocturne ou pauses respiratoires constatées par l'entourage avec reprise inspiratoire bruyante, polyurie nocturne, hypersalivation, sueurs nocturnes, éveils nocturnes multiples) ;

- un contexte clinique (obésité, hypertension artérielle, diabète de type 2, maladies cardiovasculaires, trouble de l'humeur et troubles cognitifs).

Un index d'apnées/hypopnées (IAH) supérieur ou égal à 5 par heure de sommeil confirme le diagnostic (Figure 3). La simple présence d'un IAH supérieur à 5 associée à un antécédent suscité suffit à porter le diagnostic même en l'absence de plainte diurne ou nocturne du patient [3]. En effet, le SAOS est un facteur de risque important de ces maladies [3].

La sévérité du SAOS doit être appréciée, elle permet notamment de définir les indications de prise en charge [1, 24, 26]. Elle se définit :

- $\quad$ soit par l'IAH :

○ légère : IAH entre 5 et $15 / \mathrm{h}$;

○ modérée : IAH entre 15 à $30 / \mathrm{h}$;

○ sévère : IAH supérieur à $30 / \mathrm{h}$;

- soit sur la sévérité du retentissement occasionné (somnolence diurne évaluée par l'échelle d'Epworth, somnolence au volant chez les conducteurs automobiles, sévérité de l'altération de l'architecture du sommeil, difficultés d'équilibre des pathologies associées telles que l'hypertension artérielle ou un trouble de l'humeur). 
Le plus souvent, le mécanisme des apnées et hypopnées est obstructif, définissant le SAOS, parfois il est de mécanisme central, par dysfonctionnement de la commande ventilatoire. Le SAOS est une pathologie fréquente retrouvée chez environ $5 \%$ des hommes et $3 \%$ des femmes adultes. Le facteur favorisant principal est l'obésité. Un tour de cou supérieur à $45 \mathrm{~cm}$ chez l'homme et $41 \mathrm{~cm}$ chez la femme est également un facteur favorisant. Chez le sujet non obèse, une anomalie structurelle des tissus mous ou osseux de la sphère ORL est à rechercher [3]. Le SAS central peut être associé à la respiration de Cheyne-Stroke (succession de cycles de respiration crescendo puis decrescendo séparés par des apnées centrales) et se retrouve souvent associé à l'insuffisance cardiaque congestive [3].

\subsubsection{En psychiatrie}

Le SAOS est responsable d'un retentissement sévère sur la qualité du sommeil et la qualité de vie, pouvant favoriser l'émergence ou aggraver une symptomatologie dépressive. Les études épidémiologiques retrouvent ainsi une prévalence de trouble dépressif caractérisé et de trouble bipolaire deux à trois fois supérieure qu'en population générale [23,40]. Par ailleurs, le SAOS serait particulièrement fréquent chez les sujets présentant un trouble dépressif caractérisé pharmacorésistant. En effet, il serait un facteur favorisant la pharmacorésistance et son dépistage devrait être envisagé systématiquement dans ce contexte. La prévalence du SAOS dans le trouble bipolaire est très élevée, comprise entre 20 et $40 \%$ [42]. Les outils de dépistage cliniques restent peu efficaces dans cette population et un dépistage systématique au moindre doute clinique ou en cas de pharmacorésistance est conseillé [38].

\subsubsection{Iatrogénie}

La prise de certains traitements psychotropes favorise la survenue d'un SAS, par des mécanismes indirects et directs. La prise de poids associée en particulier aux traitements antipsychotiques doit alerter le clinicien sur le risque majoré de survenue de SAOS. Ce risque est d'autant plus important en cas de co-prescription avec les benzodiazépines et apparentés. En effet, ces traitements favorisent le relâchement musculaire laryngé et la chute de la base de la langue et sont à l'origine de l'obstruction des voies aériennes supérieures pendant le sommeil. Le psychiatre doit également être vigilant quant au risque de SAS central associé 
aux prescriptions d'opioïdes de substitution, qui sont de puissants dépresseurs centraux de la ventilation nocturne.

\subsection{Les troubles moteurs nocturnes ou mouvements anormaux liés au sommeil}

\subsubsection{Généralités}

Les troubles moteurs au cours du sommeil regroupent principalement trois entités [3] :

- le syndrome des jambes sans repos (SJSR) et les mouvements périodiques des membres inférieurs (MPMI) ;

- les rythmies du sommeil ;

- le bruxisme lié au sommeil.

Leurs diagnostics restent cliniques, mais une exploration PSG est souvent justifiée pour en préciser la sévérité.

Le syndrome des jambes sans repos (SJSR) est caractérisé par la survenue de sensations désagréables des membres inférieurs (dysesthésies) accompagnées d'un besoin irrépressible de les bouger (impatiences) [24]. Ces symptômes apparaissent ou sont aggravés par l'immobilité et la position allongée, sont soulagés transitoirement par le mouvement et sont plus intenses le soir que dans la journée. Un retentissement clinique significatif est nécessaire pour porter le diagnostic de SJSR. La prévalence est d'environ $5 \%$ et le SJSR est plus fréquent chez la femme et chez les sujets de plus de 65 ans. La physiopathologie du SJSR repose sur des anomalies du transport du fer intracérébral sous-tendues par une vulnérabilité génétique, le manque de disponibilité du fer (évaluable par un dosage simple de la ferritinémie) étant responsable d'une dysrégulation du système dopaminergique (Tableau 2).

Dans $80 \%$ des cas, le SJSR est associé à la survenue de mouvements périodiques des membres inférieurs (MPMI) au cours du sommeil. Il s'agit de flexions périodiques des orteils, du pied, du genou ou de la hanche, durant quelques secondes. Ces contractions sont souvent responsables d'une fragmentation $\mathrm{du}$ sommeil. Le diagnostic des MPMI est polysomnographique, le sujet n'en ayant que rarement conscience.

Les rythmies du sommeil (ou troubles moteurs rythmiques du sommeil) sont caractérisées par des mouvements répétitifs, stéréotypés et rythmiques de grands groupes musculaires se produisant lors du sommeil ou des transitions veille-sommeil. Les rythmies les plus fréquentes sont le bercement de tout le corps (body rocking), le cognement ou le roulement de la tête (head banging ou head rolling). Ce trouble est très fréquent dans l'enfance et voit sa 
prévalence décroître rapidement avec l'âge pour ne concerner que $3 \%$ des enfants à l'âge de cinq ans. Dans de rares cas, ces troubles peuvent persister à l'âge adulte [47].

Le bruxisme lié au sommeil se caractérise par le grincement des dents ou le serrement de la mâchoire pendant le sommeil [30]. Le diagnostic de bruxisme lié au sommeil est basé sur l'anamnèse (bruits constatés par l'entourage), la présence d'usure ou de dommages dentaires, des céphalées et des douleurs orofaciales [15, 27]. Le bruxisme serait lié à une augmentation de l'activité du système nerveux autonome et des réseaux modulateurs de l'activité motrice au cours du sommeil.

\subsubsection{En psychiatrie}

Le SJSR est responsable d'un retentissement sévère sur la qualité du sommeil et la qualité de vie, pouvant favoriser l'émergence ou aggraver une symptomatologie dépressive. Les études épidémiologiques retrouvent ainsi une prévalence de trouble dépressif caractérisé deux à quatre fois supérieure qu'en population générale.

Les troubles neurodéveloppementaux (en particulier troubles du spectre de l'autisme et TDA/H) s'accompagnent régulièrement de troubles du sommeil, avec au premier plan des difficultés d'endormissement. Une fréquence élevée de troubles moteurs rythmiques est observée dans cette population. Ceux-ci doivent cependant être distingués des comportements moteurs stéréotypés survenant en veille.

Enfin, les troubles anxieux seraient largement associés au bruxisme lié au sommeil (principalement chez l'enfant), probablement par un mécanisme d'hyperactivation neurovégétative.

\subsubsection{Iatrogénie}

La prise de certains traitements psychotropes s'accompagne de la survenue de troubles moteurs liés au sommeil, en particulier les antidépresseurs et les antipsychotiques. Un SJSR peut apparaître ou être aggravé par la prise d'antidépresseurs, en particulier les antidépresseurs noradrénergiques et sérotoninergiques spécifiques (NaSSA), comme la mirtazapine. L'utilisation de médication antipsychotique, en particulier de première génération, est également associée à la survenue de SJSR; ces manifestations doivent cependant être distinguées des akathisies, bien que les deux syndromes soient fréquemment 
comorbides (Tableau 3). Le bruxisme lié au sommeil peut également survenir ou être aggravé suite à la prise de certains antidépresseurs, en particulier sérotoninergiques.

\subsection{Les troubles comportementaux du sommeil ou parasomnies}

\subsubsection{Généralités}

Les parasomnies regroupent une série de troubles du sommeil caractérisés par la survenue de manifestations comportementales indésirables au cours du sommeil [45]. On distingue habituellement [3] :

- les parasomnies survenant en sommeil lent profond (somnambulisme, éveils confusionnels et terreurs nocturnes);

- les parasomnies survenant en sommeil paradoxal (troubles du comportement en sommeil paradoxal, maladies des cauchemars).

\subsubsection{Les parasomnies du sommeil lent profond}

Les parasomnies du sommeil lent profond surviennent principalement dans le premier tiers de la nuit et sont accompagnées d'une amnésie partielle ou complète. Elles concernent volontiers les enfants et les adultes jeunes et sont liées à des troubles l'éveil et à une dissociation entre les états de conscience de veille et de sommeil [3] (Figure 4).

Dans le somnambulisme, les manifestations comportementales motrices sont au premier plan, caractérisées par des déambulations et des comportements moteurs maladroits, complexes, d'allure automatique. Les terreurs nocturnes sont caractérisées par l'importance des manifestations comportementales et neurovégétatives de peur (tachycardie, hypersudation, tachypnée, mydriase). Enfin, les éveils confusionnels se distinguent par la faible composante motrice et neurovégétative au cours de l'épisode, ils s'accompagnent très souvent de somniloquie. Les accès peuvent être plus longs et des comportements complexes peuvent être observés (Tableau 4).

Le retentissement des parasomnies du sommeil lent profond doit faire l'objet d'une évaluation systématique. Il est marqué par un risque de mise en danger, de blessures du sujet lui-même ou de son partenaire de lit, de somnolence diurne excessive et gêne sociale [31, 32].

Si les parasomnies du sommeil lent profond sont associées à une fréquence plus élevée de symptômes anxieux et dépressifs, elles ne constituent pas l'expression d'une pathologie psychiatrique sous-jacente.

Leur diagnostic est clinique, mais la réalisation d'une PSG est indiquée afin [45] : 
- d'éliminer un diagnostic différentiel (principalement épilepsie frontale nocturne) ;

- lorsque le retentissement diurne (somnolence) est important, justifiant la recherche d'autres troubles du sommeil associés, pouvant aussi favoriser les parasomnies du sommeil lent profond.

La PSG révèle alors, outre l'objectivation de comportements complexes en sommeil lent profond, une fragmentation excessive de celui-ci (Figure 4).

\subsubsection{Les parasomnies du sommeil paradoxal}

Le trouble du comportement en sommeil paradoxal correspond à une agitation au cours du sommeil liée à la mise en acte des rêves du sujet. Elle survient le plus souvent en deuxième partie de nuit et est liée à la perte de l'atonie musculaire normale du sommeil paradoxal. Le sujet présente des comportements moteurs plus ou moins élaborés, avec des mouvements brusques, parfois violents, pouvant être responsables de chutes du lit, de blessures pour le patient lui-même ou son partenaire. Cette parasomnie concerne principalement le sujet âgé de sexe masculin, elle est fortement associée aux maladies neurodégéneratives de type maladie de Parkinson et autres synucléopathies dont elle constitue le plus souvent un signe prodromique. Son diagnostic doit être confirmé par un enregistrement PSG, l'enregistrement révélant, outre des comportements moteurs complexes en sommeil paradoxal, une activité phasique musculaire anormalement élevée au cours de ce dernier.

\subsubsection{En psychiatrie}

L'agitation au cours du sommeil n'est pas rare chez les patients souffrant de troubles psychiatriques, bien qu'elle ne soit que très rarement rapportée de façon spontanée. Elle peut constituer une manifestation spécifique du trouble psychiatrique, comme au cours de l'état de stress post-traumatique (ESPT), ou être d'origine iatrogène. Les troubles du sommeil sont au premier plan dans l'ESPT. Ils résultent d'un état d'hypervigilance (responsable d'une insomnie) et de symptômes de reviviscence (responsables de cauchemars post-traumatiques et d'agitation nocturne). Les cauchemars post-traumatiques se distinguent des cauchemars dits «idiopathiques » par leur caractère répétitif et la reproduction exacte ou symbolique de l'événement traumatique. L'agitation nocturne au cours de l'ESPT, bien que très fréquente, est un symptôme très rarement étudié, dont la physiopathologie reste mal comprise. Des travaux suggèrent l'implication d'une dysrégulation du sommeil paradoxal, avec une perte de 
l'atonie, tendant à rapprocher ce phénomène du TCSP. La prazosine constitue un traitement de premier ordre pour les parasomnies spécifiquement associées à l'ESPT.

Les diagnostics différentiels psychiatriques des troubles comportementaux du sommeil sont : les attaques de panique nocturnes qui se produisent pendant ou immédiatement après des réveils nocturnes, généralement en sommeil lent, et les troubles dissociatifs liés au sommeil, qui partagent les caractéristiques des troubles dissociatifs avec déréalisation et dépersonnalisation, pendant ou immédiatement après des réveils nocturnes.

\subsubsection{Iatrogénie}

La survenue d'épisodes d'agitation nocturne chez des sujets recevant une médication psychotrope doit faire poser la question d'une origine iatrogène [39]. L'utilisation d'hypnotiques à demi-vie courte (Zolpidem, Zopiclone) est très associée à la survenue d'éveils confusionnels. Une fréquence élevée de somnambulisme est rapportée chez les patients recevant un traitement par lithium. Enfin, il existe une littérature grandissante sur l'association entre la prise d'antidépresseurs et la survenue de troubles du comportement en sommeil paradoxal.

\subsection{Hypersomnolence et hypersomnies centrales}

\subsubsection{Généralités}

La somnolence diurne se définit comme l'incapacité à rester éveillé et alerte pendant les périodes de veille de la journée. Le sommeil permet de réduire la somnolence diurne. Elle doit être distinguée de la fatigue [22]. La fatigue est l'épuisement progressif des performances physiques et cognitives associées à une charge de travail accrue. Le repos permet de réduire la fatigue. La somnolence diurne est un état subjectif et objectif d'éveil physiologique abaissé avec incapacité à se maintenir éveillé.

Le terme d'hypersomnolence est utilisé pour décrire les symptômes liés à une somnolence diurne. Le terme d'hypersomnie est utilisé pour décrire une maladie spécifique liée à une hypersomnolence d'origine centrale.

L'hypersomnolence est une plainte fréquente avec $15 \%$ de la population présentant une somnolence modérée et 4-5\% une somnolence sévère. Elle représente un enjeu de santé publique du fait du risque accidentologique associé. L'hypersomnolence revêt de ce fait des 
implications médico-légales, elle fait partie de la liste des affections médicales incompatibles avec l'obtention ou le maintien du permis de conduire [37].

L'hypersomnolence est multifactorielle, pouvant être la conséquence [3] :

- d'un trouble du sommeil (en particulier respiratoire avec le SAOS), on parle d'hypersomnolence secondaire à un trouble du sommeil ;

- d'une insuffisance chronique de sommeil ;

- de pathologie neurologique, endocrinienne ou psychiatrique: on parle d'hypersomnolence secondaire à une pathologie médicale ;

- d'une consommation de psychotrope ou de son sevrage : on parle d'hypersomnolence secondaire à l'usage d'une substance ;

- enfin d'une hypersomnie centrale qui sont principalement la narcolepsie (type 1 et 2) et l'hypersomnie idiopathique [17,28].

Ces hypersomnies centrales sont des troubles du sommeil rares regroupant des entités nosographiques caractérisées par la présence au premier plan d'une hypersomnolence.

La narcolepsie type 1 est caractérisée par l'association :

- d'une hypersomnolence (sévère, caractérisée par des accès de sommeil diurnes multiples et courts, incoercibles, rafraichissants, souvent accompagnés d'activité onirique) ;

- de cataplexies (abolition brève et brutale du tonus musculaire, partielle ou généralisée, sans altération de la conscience, déclenchées par une émotion le plus souvent positive) ;

- des symptômes associés à une dysrégulation du sommeil paradoxal, comme les hallucinations liées au sommeil (hypnagogiques à l'endormissement ou hypnopompiques au réveil), les paralysies de sommeil (paralysie complète et transitoire, durant quelques secondes à quelques minutes survenant au moment de l'endormissement ou du réveil) et le trouble du comportement en sommeil paradoxal [18].

La narcolepsie type 1 est associée à une perte de fonction des neurones à hypocrétine1/orexine-A. La narcolepsie type 2 est caractérisée sur le plan clinique par une hypersomnolence sans cataplexie, associée ou non à d'autres signes cliniques de dysrégulation du sommeil paradoxal [7]. La présence d'au moins deux endormissements en sommeil paradoxal (à la fois sur la PSG et les cinq tests diurnes du TILE), associés à une latence moyenne d'endormissement inférieure à huit minutes est un élément essentiel du diagnostic de narcolepsie. 
L'hypersomnie idiopathique est principalement caractérisée par :

- un allongement du temps de sommeil sur $24 \mathrm{~h}$;

- une importante inertie au réveil, pouvant aller jusqu'à une ivresse du réveil avec confusion ;

Une hypersomnolence est sévère, continue et présente dès le réveil.

Contrairement à la narcolepsie, les accès de sommeil diurnes sont souvent longs (plusieurs heures) et non restaurateurs de vigilance normale. Le temps total de sommeil est supérieur à 11/24 heures et, contrairement à la narcolepsie, d'excellente qualité, avec une efficacité souvent supérieure à $90 \%$ [10].

Le diagnostic des hypersomnies centrales est établi selon des critères cliniques, neurophysiologiques (PSG et TILE) et parfois biologiques et nécessite des évaluations dans les centres de référence.

\subsubsection{En psychiatrie}

Dans le trouble dépressif caractérisé, les symptômes d'hypersomnolence sont rapportés avec une fréquence estimée entre $9 \%$ chez les enfants et $76 \%$ chez les adultes jeunes, avec une nette prédominance féminine. Trois conditions cliniques méritent une attention particulière :

- dans le trouble dépressif caractérisé avec caractéristiques atypiques, l'hypersomnolence constitue l'un des critères diagnostiques, avec l'hyperréactivité émotionnelle, l'augmentation d'appétit accompagnée ou non de prise de poids, les sensations de lourdeur dans les membres et l'hypersensibilité au rejet. Pourtant, les symptômes d'hypersomnolence ne sont retrouvés que dans 24 à 56 \% des cas ;

- au cours du trouble affectif saisonnier, les symptômes d'hypersomnolence sont plus constants, avec une fréquence rapportée comprise entre 67 à $76 \%$;

- enfin, les épisodes dépressifs caractérisés au cours du trouble bipolaire de type 2 sont plus fréquemment associés à une hypersomnolence que ceux survenant dans le cadre d'un trouble dépressif récurrent.

Les disparités de fréquence observées entre les études semblent principalement expliquées par des considérations méthodologiques, avec une définition de l'hypersomnolence variable selon les études [20]. Au cours du trouble dépressif caractérisé, la plainte d'hypersomnolence est rarement corroborée par des mesures objectives de la somnolence comme le TILE. Par 
ailleurs, l'hypersomnolence est souvent associée à un sommeil de mauvaise qualité et une fluctuation d'un jour à l'autre.

Il n'existe pas à ce jour de recommandations spécifiques pour la prise en charge de l'hypersomnolence dans le trouble dépressif caractérisé. Des recommandations simples peuvent être formulées, comme limiter l'utilisation de psychotropes à propriétés sédatives comme les benzodiazépines et favoriser celle d'antidépresseurs noradrénergiques pour leurs propriétés stimulantes. La prescription de molécules psychostimulantes n'est pas recommandée en première ligne. La persistance d'une hypersomnolence malgré une prise en charge adéquate des autres symptômes dépressifs doit conduire à réaliser une polysomnographie avec TILE afin de ne pas méconnaître une hypersomnie centrale sousjacente. Les hypersomnies centrales sont en effet fréquemment associées à des symptômes dépressifs, en particulier au cours de la narcolepsie type 1.

Les sujets souffrant de trouble déficit de l'attention/hyperactivité rapportent fréquemment une hypersomnolence, particulièrement dans les situations d'inactivité. Cette propension à la somnolence diurne est confirmée par des mesures objectives, à la fois chez les enfants et les adultes. Les troubles du sommeil, en particulier l'insomnie, le syndrome de retard de phase du sommeil ou le syndrome des jambes sans repos sont particulièrement prévalents au cours de ce trouble, pourtant la présence d'une hypersomnolence n'apparaît pas être systématiquement la conséquence du mauvais sommeil de nuit.

L'hypothèse d'une dysrégulation des systèmes de l'éveil dans le TDAH est désormais appuyée par de nombreuses études [13,36]. L'inattention et l'impulsivité pourraient constituer des conséquences cognitives de la baisse de vigilance, le sujet développant des symptômes d'hyperactivité pour y faire face. Si le TDAH partage les mêmes thérapeutiques (méthylphénidate, modafinil) avec les hypersomnies centrales, il pourrait donc en partager également les mécanismes physiopathologiques. L'évaluation clinique du TDAH doit comprendre une mesure subjective de la somnolence (Echelle d'Epworth) et être complétée par une polysomnographie avec TILE en cas de plainte cliniquement significative.

Enfin, il faut noter que la plainte dans le cadre des troubles somatoformes (comme le syndrome de fatigue chronique) correspond plus généralement à de la fatigue qu'à une hypersomnolence [35].

\subsubsection{Iatrogénie}


Les patients souffrant de pathologies psychiatriques sont particulièrement vulnérables à la survenue d'une hypersomnolence liée à l'usage de substances. Elle peut être de trois types [3] :

- hypersomnolence secondaire à l'utilisation de psychotropes sédatifs (benzodiazépines, hypnotiques, antipsychotiques, antiépileptiques, opiacés, antihistaminiques, antidépresseurs sédatifs), en particulier en cas d'associations médicamenteuses ;

- hypersomnolence secondaire à l'usage de substances (alcool, opiacés, cannabis) ;

- hypersomnolence secondaire à un sevrage de psychostimulants (médicamenteux ou illicites).

\section{Conclusion}

Les troubles du sommeil font partie de la pratique clinique quotidienne du psychiatre [6,9]. Les explorations électrophysiologiques du sommeil sont des examens d'intérêt diagnostique dans l'évaluation des comorbidités associées aux troubles psychiatriques. Les pathologies du sommeil ont un impact important sur le pronostic du trouble psychiatrique initial, et plus généralement sur le pronostic en terme de morbi-mortalité et de qualité de vie [6,48]. Lorsque l'on reste attentif au vécu du patient, ces explorations du sommeil ne sont pas plus difficiles à réaliser chez les sujets souffrant de troubles psychiatriques qu'en population générale. Une collaboration étroite entre médecine du sommeil et psychiatrie est nécessaire afin de favoriser l'accès, pour les sujets souffrant de trouble psychiatrique, aux explorations du sommeil, dans l'objectif de leur assurer une prise en charge de qualité.

Conflit d'intérêt : aucun 


\section{Figure 1}

Descriptions des explorations du sommeil traditionnelles

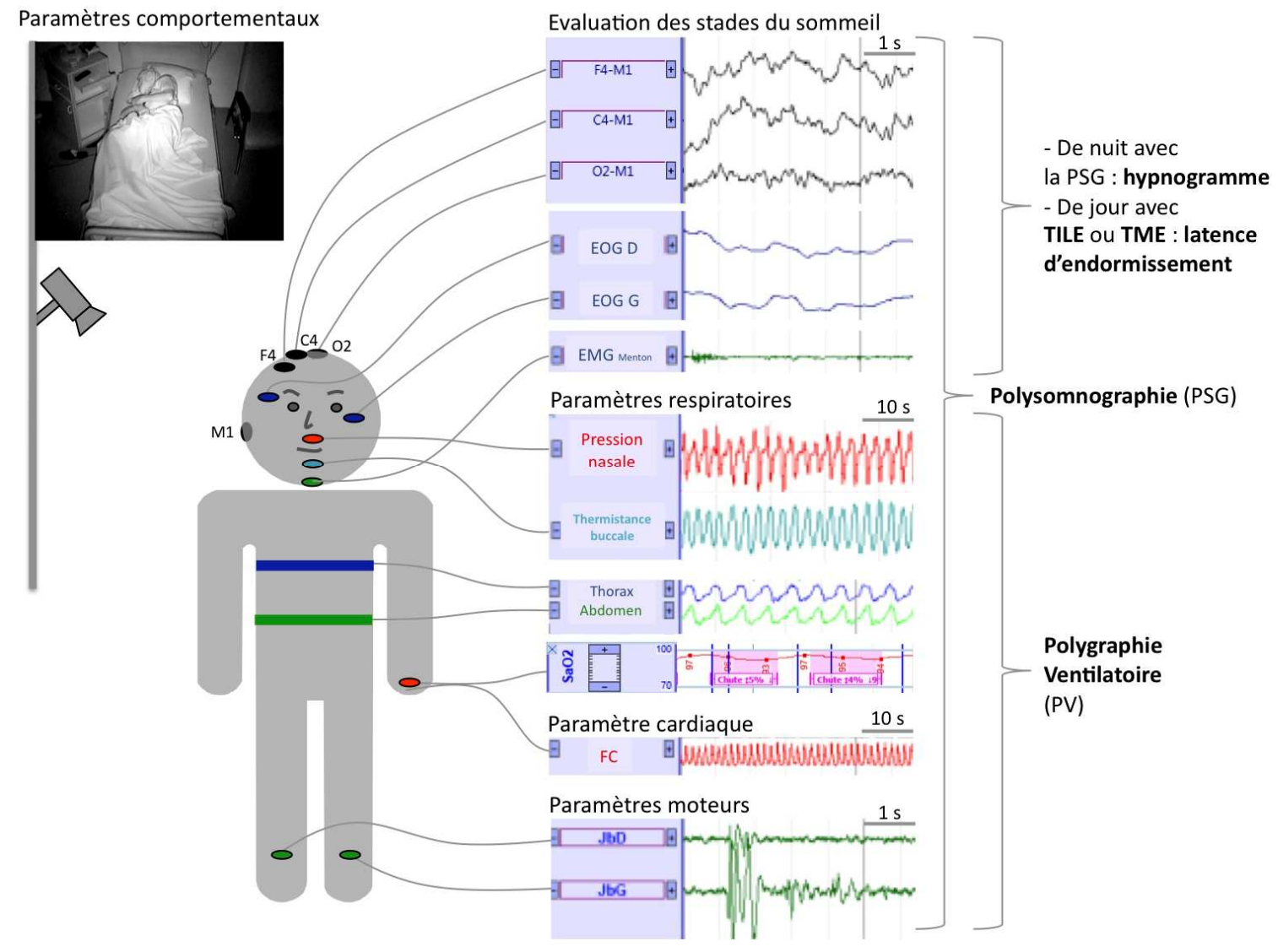




\section{Figure 2}

Les différents stades du sommeil
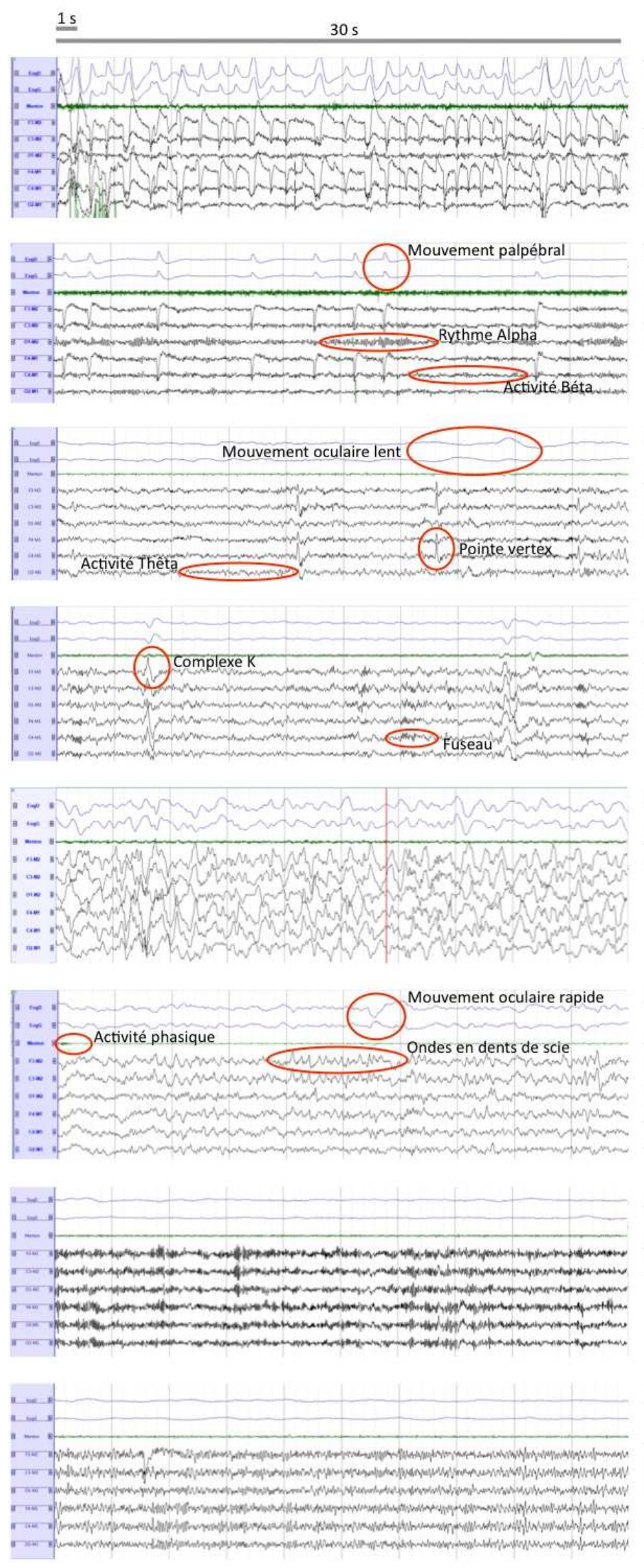

\section{Veille active (W)}

Artéfacts musculaires

Nombreux mouvements oculaires et

palpébraux

\section{Veille calme (W) \\ Rythme Alpha de fond \\ Activité dans la bande Béta \\ à l'ouverture des yeux}

\section{Stade N1 (NREM 1)}

Activité de fond mixte Mouvements oculaires lents Pointe vertex

\section{Stade N2 (NREM 2) \\ Activité de fond mixte \\ Complexes $\mathrm{K}$ \\ Fuseaux (Spindle)}

\section{Stade N3 (NREM 3)}

Activité de fond lente dans la bande Delta Possibles fuseaux

\section{Stade R (REM)}

Activité de fond mixte

Ondes en dents de scie

Mouvements oculaires rapides

Activité musculaire tonique abolie

Activités musculaires phasiques brèves

\section{Stade N1}

Activités rapides induites par la prise de Benzodiazépine (Diazépam dans ce cas)

\section{Stade N1}

Activités paroxystiques polymorphes induites par la prise de lithium ou antipsychotique (Lithium et Olanzapine dans ce cas) 


\section{Figure 3}

Les événements respiratoires ou moteurs pathologiques

$10 \mathrm{~s}$

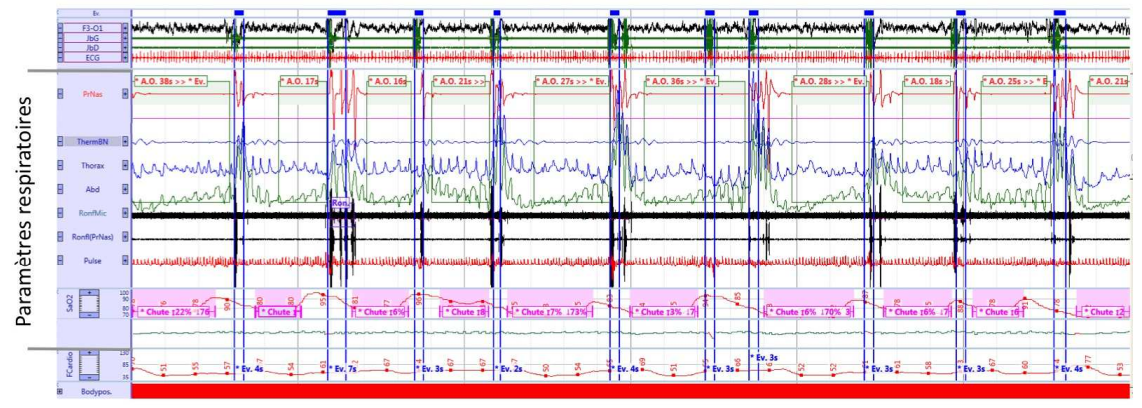

Syndrome d'Apnées Obstructives du Sommeil

Arrêt du débit respiratoire

pendant plus de $10 \mathrm{~s}$

Avec persistance des efforts

respiratoires

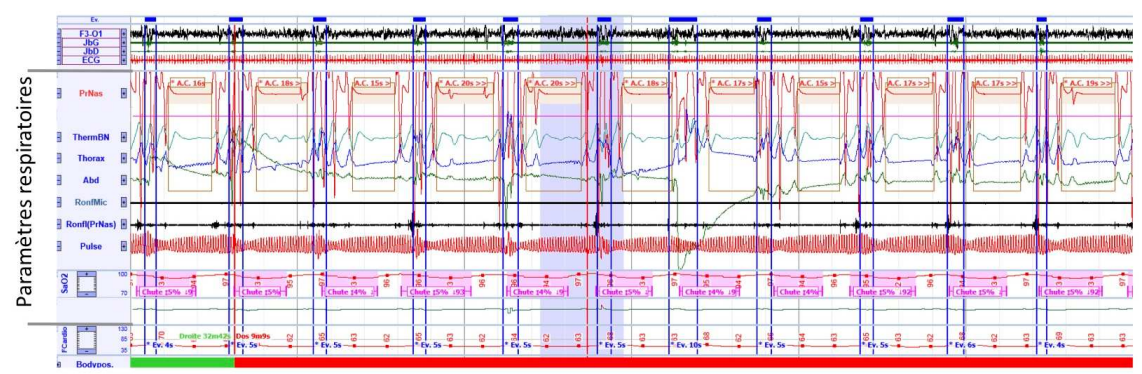

\section{Syndrome d'Apnées} Centrales du Sommeil

Arrêt du débit respiratoire pendant plus de $10 \mathrm{~s}$

Avec disparition des efforts respiratoires

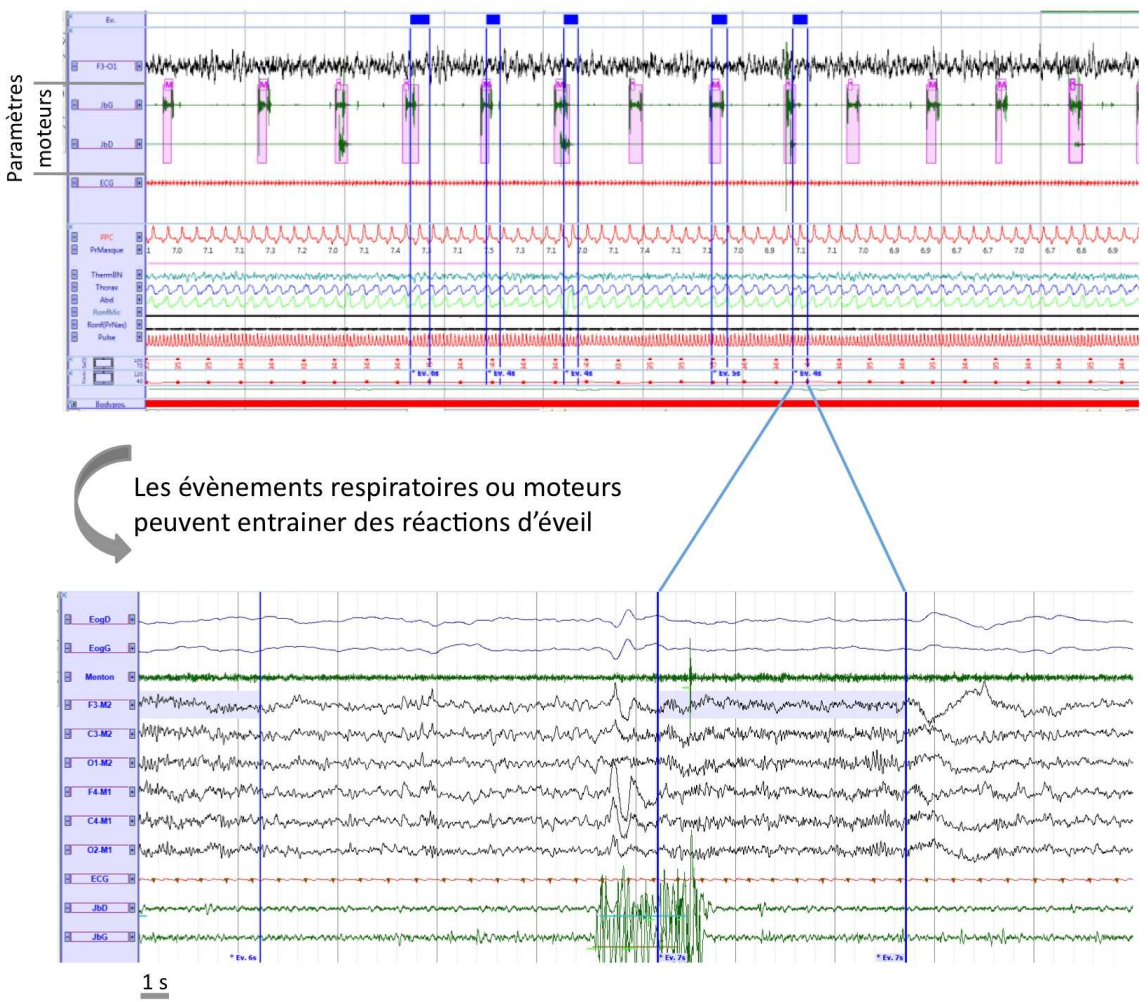

\section{Syndrome des} mouvements périodiques de jambes

Mouvements périodiques des membres inférieurs

\section{Réaction d'éveil}

Provoquée par une apnée ou un mouvement périodique des membres inférieurs 


\section{Figure 4}

Les parasomnies du sommeil lent profond
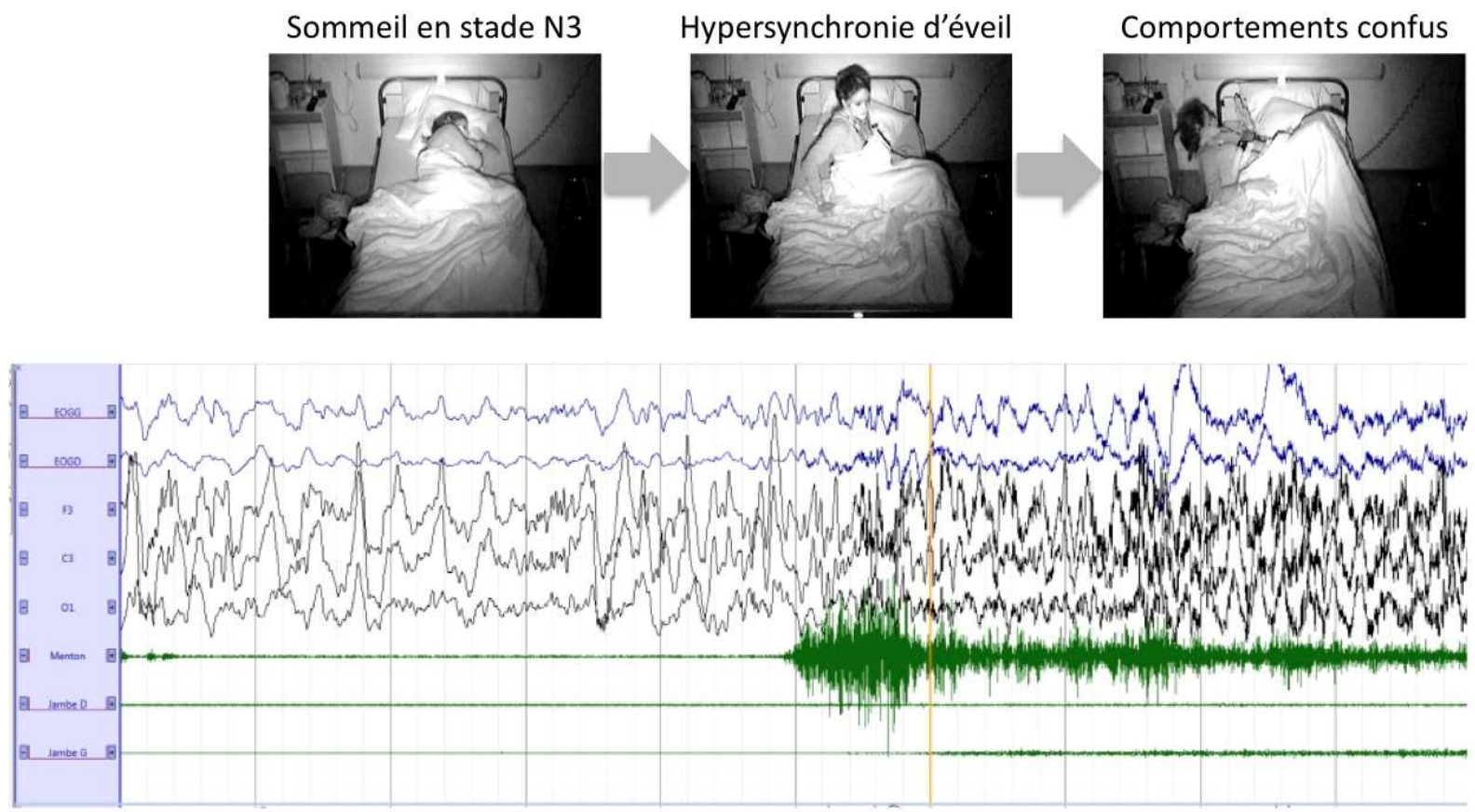
Tableau 1 : L'Échelle d'Epworth pour évaluer la somnolence diurne sévère

\begin{tabular}{lllll}
\hline Situation & \multicolumn{3}{l}{ Chance de s'assoupir } \\
\hline Assis, en train de lire & 0 & 1 & 2 & 3 \\
En train de regarder la télévision & 0 & 1 & 2 & 3 \\
Assis, inactif, dans un endroit public (au théâtre, en & 0 & 1 & 2 & 3 \\
réunion) & & & & \\
Comme passager dans une voiture roulant sans arrêt & 0 & 1 & 2 & 3 \\
$\begin{array}{l}\text { pendant une heure } \\
\text { Allongé l'après-midi pour se reposer quand les }\end{array}$ & 0 & 1 & 2 & 3 \\
circonstances le permettent & & & & \\
Assis en train de parler à quelqu'un & 0 & 1 & 2 & 3 \\
Assis calmement après un repas sans alcool & 0 & 1 & 2 & 3 \\
Dans une auto immobilisée quelques minutes dans un & 0 & 1 & 2 & 3 \\
encombrement & & & & \\
\end{tabular}

Instructions : Vous arrive-t-il de somnoler ou de vous endormir, et non de vous sentir seulement fatigué, dans les situations suivantes ? Cette question concerne votre vie dans les mois derniers. Même si vous ne vous êtes pas trouvé récemment dans l'une des situations suivantes, essayez de vous représenter comment elles auraient pu vous affecter.

Choisissez dans l'échelle suivante le chiffre le plus approprié à chaque situation. $0=$ ne s'assoupirai jamais ; 1 = faible chance de s'assoupir ; $2=$ chance moyenne de s'assoupir ; $3=$ forte chance de s'assoupir. 
Tableau 2 : Les quatre critères diagnostiques essentiels du syndrome des jambes sans repos

\section{Critères}

1 Besoin impérieux de bouger les membres (impatience motrice), généralement causé par des sensations désagréables ou inconfortables dans les jambes.

2 L'envie de bouger et les symptômes désagréables sont exclusivement présents au repos (c'est-à-dire en position allongé(e), assise)) ou accentués, avec au moins une amélioration temporaire ou partielle, par l'activité.

3 L'envie de bouger et les sensations désagréables (dysesthésies) s'aggravent le soir ou la nuit.

4 L'envie de bouger et les sensations désagréables sont soulagées partiellement ou totalement par le mouvement. 
Tableau 3 : Akathisie et Syndrome des Jambes Sans Repos (SJSR)

\begin{tabular}{|c|c|c|}
\hline & Akathisie & SJRS \\
\hline Impatience & $\begin{array}{l}\text { Non limité aux jambes } \\
\text { Associé à impatience psychologique } \\
\text { (tension interne, sentiment } \\
\text { d'agitation) }\end{array}$ & $\begin{array}{l}\text { Limité aux jambe mais pouvant } \\
\text { s'étendre aux membres supérieurs }\end{array}$ \\
\hline $\begin{array}{l}\text { Aggravé par le } \\
\text { repos }\end{array}$ & $+/-$ & +++ \\
\hline $\begin{array}{l}\text { Soulagé par le } \\
\text { mouvement }\end{array}$ & $+/-$ & +++ \\
\hline $\begin{array}{l}\text { Caractère } \\
\text { vespéral }\end{array}$ & - & +++ \\
\hline $\begin{array}{l}\text { Syndrome } \\
\text { extrapyramidal } \\
\text { associé }\end{array}$ & ++ & - \\
\hline $\begin{array}{l}\text { Induit par } \\
\text { neuroleptiques }\end{array}$ & +++ & +++ \\
\hline $\begin{array}{l}\text { Induit par } \\
\text { antidépresseurs }\end{array}$ & + & ++ \\
\hline
\end{tabular}


Tableau 4 : Les parasomnies du sommeil lent profond

\begin{tabular}{llll}
\hline & Éveil confusionnel & Terreur nocturne & Somnambulisme \\
\hline Comportements & $+/-$ & $+/-$ & +++ \\
complexes & & & \\
Niveau & $+/-$ & +++ & $+/-$ \\
végétatif & & & \\
\hline
\end{tabular}




\section{Références}

[1] Adult Obstructive Sleep Apnea Task Force of SPLF, Société française de recherche et de médecine du sommeil, Société française d'ORL, Société française de médecine du travail, Société de physiologie, Société française de cardiologie, et al. Synthèse des recommandations de bonne pratique clinique : prise en charge du syndrome d'apnéeshypopnées de l'adulte obstructives du sommeil (SAHOS). Médecine du Sommeil 2010;7:161-65.

[2] American Academy of Sleep Medicine The AASM Manual for the Scoring of Sleep and Associated Events. 2015.

[3] American Academy of Sleep Medicine, Société Française de Recherche et Médecine du Sommeil. Classification Internationale des Pathologies du Sommeil, $3^{\mathrm{e}}$ version. 2014.

[4] Arand D, Bonnet M, Hurwitz T, Mitler M, Rosa R, Sangal RB. The clinical use of the MSLT and MWT. Sleep 2005;28:123-44.

[5] Arnulf I, Vecchierini M, Rey M. Recommandations de la Société française de recherche et de médecine du sommeil (SFRMS). Procédure de réalisation des tests itératifs de vigilance. Médecine du Sommeil 2008;5:38-41.

[6] Bassetti CL, Ferini-Strambi L, Brown S, Adamantidis A, Benedetti F, Bruni O, et al. Neurology and psychiatry: Waking up to opportunities of sleep. : State of the art and clinical/research priorities for the next decade. Eur J Neurol 2015;22:1337-54.

[7] Baumann CR, Mignot E, Lammers GJ, Overeem S, Arnulf I, Rye D, et al. Challenges in diagnosing narcolepsy without cataplexy: A consensus statement. Sleep 2014;37:1035-42.

[8] Besset A. Exploration de la somnolence. Médecine du Sommeil 2004;1:15-21.

[9] Billiard M, Dauvilliers Y. Les troubles du sommeil. Paris: Elsevier-Masson; 2012.

[10] Billiard M, Sonka K. Idiopathic hypersomnia. Sleep medicine reviews 2015; In press

[11] Brion A, Pallanca O. Polysomnographie en psychiatrie. In: Vion-Dury J, Balzani C, Micoulaud Franchi JA, eds. Neurophysiologie clinique en psychiatrie. Paris: ElsevierMasson; 2015. p. 105-32

[12] Claustrat B. Mélatonine et troubles du rythme veille-sommeil. Médecine du Sommeil 2009;6:12-24.

[13] Collop NA, Anderson WM, Boehlecke B, Claman D, Goldberg R, Gottlieb DJ, et al. Clinical guidelines for the use of unattended portable monitors in the diagnosis of obstructive sleep apnea in adult patients. Portable Monitoring Task Force of the American Academy of Sleep Medicine. J Clin Sleep Med 2007;3:737-47.

[14] Collop NA, Tracy SL, Kapur V, Mehra R, Kuhlmann D, Fleishman SA, et al. Obstructive sleep apnea devices for out-of-center (OOC) testing: technology evaluation. J Clin Sleep Med 2011;7:531-48.

[15] D'Incau E, Brocard D, Laluque J Lésions d'usure et bruxismes chez l'adulte. Revue d'Odonto-Stomatologie 2014;43:308-24.

[16] Daston L, Galison P. Objectivité. Dijon: Les Presses du réel; 2012.

[17] Dauvilliers Y, Arnulf I. Narcolepsy with cataplexy. Rev Neurol (Paris) 2008;164:63445.

[18] Dauvilliers Y, Arnulf I, Mignot E. Narcolepsy with cataplexy. Lancet 2007;369:499511.

[19] Dauvilliers Y, Billiard M. Aspects du sommeil normal. EMC-Neurologie. Vol. 17-025A-10. Paris: Elsevier-Masson; 2004.

[20] Dauvilliers Y, Lopez R, Ohayon M, Bayard S. Hypersomnia and depressive symptoms: methodological and clinical aspects. BMC Med 2013;11:78. 
[21] Gibbs F, Gibbs E. Atlas of Electroencephalography. Cambridge: Lew A. Cummings Co; 1941.

[22] Haba-Rubio J, Krieger J. Somnolence, fatigue et hypersomnie. Médecine du Sommeil 2011;8:5-14.

[23] Harris M, Glozier N, Ratnavadivel R, Grunstein RR. Obstructive sleep apnea and depression. Sleep Med Rev 2009;13:437-44.

[24] Karroum E, Konofal E, Arnulf I. Restless-legs syndrome. Rev Neurol (Paris) 2008;164:701-21.

[25] Kushida CA, Littner MR, Morgenthaler T, Alessi CA, Bailey D, Coleman J, Jr, et al. Practice parameters for the indications for polysomnography and related procedures: an update for 2005. Sleep 2005;28:499-521.

[26] Launois S, Rey M. Place des différents enregistrements au cours du sommeil : résumé du rapport d'évaluation de l'HAS. Médecine du Sommeil 2013;10:6-11.

[27] Lavigne GJ, Khoury S, Abe S, Yamaguchi T, Raphael K Bruxism physiology and pathology: an overview for clinicians. J Oral Rehabil 2008;35:476-94.

[28] Lecendreux M, Dauvilliers Y, Arnulf I, Franco P. Narcolepsy with cataplexy in the child: clinical evaluation and therapeutical management. Rev Neurol (Paris) 2008;164:646-57.

[29] Littner MR, Kushida C, Wise M, Davila DG, Morgenthaler T, Lee-Chiong T, et al. Practice parameters for clinical use of the multiple sleep latency test and the maintenance of wakefulness test. Sleep 2005;28:113-21.

[30] Lobbezoo F, Ahlberg J, Glaros AG, Kato T, Koyano K, Lavigne GJ, et al. Bruxism defined and graded: an international consensus. J Oral Rehabil 2013;40:2-4.

[31] Lopez R, Jaussent I, Dauvilliers Y Objective daytime sleepiness in patients with somnambulism or sleep terrors. Neurology 2014;83:2070-6.

[32] Lopez R, Jaussent I, Scholz S, Bayard S, Montplaisir J, Dauvilliers Y. Functional impairment in adult sleepwalkers: a case-control study. Sleep 2013;36:345-51.

[33] Micoulaud-Franchi JA, Balzani C, Faugere M, Cermolaccce M, Naudin J, Vion-Dury J. Clinical neurophysiology in psychiatry: 1 - Techniques, vocabularies and indications of conventional electroencephalogram. Ann Med Psychol 2013;171:334-41.

[34] Micoulaud-Franchi JA, Geoffroy PA, Amad A, Quiles C. Le jardinier et le botaniste. Proposition d'une organiation minimale de la sémiologie psychiatrique pour l'étudiant en médecine. Ann Med Psychol 2015;173:460-69.

[35] Micoulaud-Franchi JA, Hingray C, Gerardin P, Bertschy G. Troubles somatoformes à tous les âges. La revue du praticien. In Press;

[36] Morgenthaler TI, Lee-Chiong T, Alessi C, Friedman L, Aurora RN, Boehlecke B, et al. Practice parameters for the clinical evaluation and treatment of circadian rhythm sleep disorders. An American Academy of Sleep Medicine report. Sleep 2007;30:1445-59.

[37] Philip P, Chaufton C. Quel est l'intérêt du test de maintien de l'éveil pour déterminer l'aptitude à la conduite chez les patients somnolents ? Médecine du Sommeil 2013;10:167-70.

[38] Plante DT, Winkelman JW. Sleep disturbance in bipolar disorder: therapeutic implications. Am J Psychiatry 2008;165:830-43.

[39] Pressman MR. Factors that predispose, prime and precipitate NREM parasomnias in adults: clinical and forensic implications. Sleep Med Rev 2007;11:5-30; discussion 31-3.

[40] Schroder CM, O'Hara R. Depression and Obstructive Sleep Apnea (OSA). Ann Gen Psychiatry 2005;4:13. 
[41] Silber MH, Ancoli-Israel S, Bonnet MH, Chokroverty S, Grigg-Damberger MM, Hirshkowitz M, et al. The visual scoring of sleep in adults. J Clin Sleep Med 2007;3:121-31.

[42] Soreca I, Buttenfield JA, Hall MH, Kupfer DJ. Screening for obstructive sleep apnea in patients with bipolar I disorder: comparison between subjective and objective measures. Bipolar Disord 2014;17:345-8.

[43] Stal V Nuits mouvementées et bruyantes : comment différencier parasomnies et crises d'épilepsie frontale nocturnes ? Intérêt de l'enregistrement vidéo-EEGpolysomnographique. Médecine du Sommeil 2009;6:65-73.

[44] Taillard J. Procédures de réalisation des tests de maintien d'éveil et valeurs normatives: Recommandations des bonnes pratiques cliniques. Société française de recherche et médecine du sommeil (SFRMS), mise à jour 2014. Médecine du Sommeil 2013;11:206-8.

[45] Vecchierini M. Quand et comment explorer une parasomnie? Médecine du Sommeil 2005;1:33-40.

[46] Vespignani H. L'EEG : De la technique à la clinique. Parus: John Libbey Eurotext. 2003.

[47] Vitello N, Bayard S, Lopez R, Carlander B, Dauvilliers Y, Cochen De Cock V. Rhythmic movement disorder associated with restless legs syndrome. Sleep Med 2012;13:1324-5.

[48] Wykes T, Haro JM, Belli SR, Obradors-Tarrago C, Arango C, Ayuso-Mateos JL, et al. Mental health research priorities for Europe. Lancet Psychiatry 2015. 